\title{
Correction to: Non-contrast MRI for breast screening: preliminary study on detectability of benign and malignant lesions in women with dense breasts
}

\author{
Yangyang $\mathrm{Bu}^{1,2}$. Jun Xia ${ }^{3} \cdot$ Bobby Joseph ${ }^{4} \cdot$ Xianjing Zhao ${ }^{1,2} \cdot$ Maosheng $\mathrm{Xu}^{1,2} \cdot{\text { Yingxing } \mathrm{Yu}^{1,2}}^{1}$. Shouliang $\mathrm{Qi}^{5}$. \\ Kamran A. Shah ${ }^{4}$. Shiwei Wang ${ }^{1,2}$. Jiani Hu ${ }^{4}$ (D)
}

Published online: 20 August 2019

๑) Springer Science+Business Media, LLC, part of Springer Nature 2019

\section{Correction to: Breast Cancer Research and Treatment https://doi.org/10.1007/s10549-019-05342-5}

In the original version of the article, the image of Fig. 2 was erroneously duplicated as Fig. 4. The correct version of Fig. 4 is given below. The original article has been corrected.

The original article can be found online at https://doi.org/10.1007/ s10549-019-05342-5.

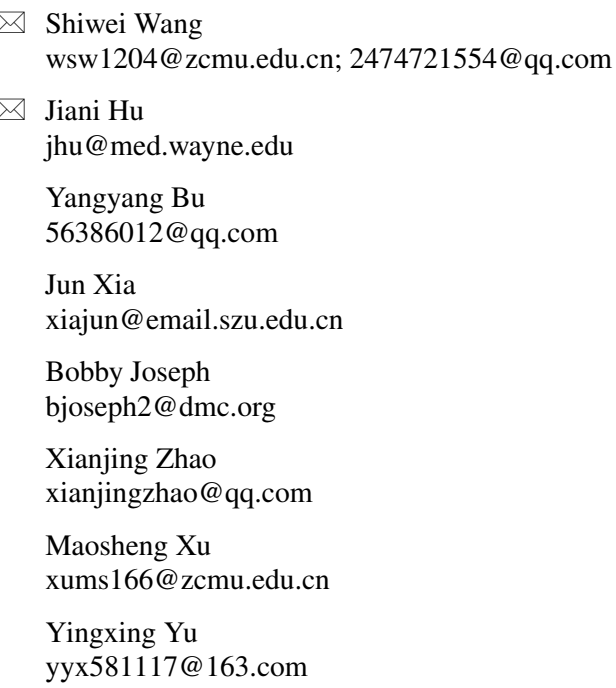

Shouliang Qi qis1@bmie.neu.edu.cn

Kamran A. Shah kshah@wayne.edu

1 The First Clinical Medical College, Zhejiang Chinese Medical University, Hangzhou 310053, China

2 Department of Radiology, The First Affiliated Hospital of Zhejiang Chinese Medical University, 54 Youdian Road, Hangzhou 310006, China

3 Department of Radiology, The First Affiliated Hospital of Shenzhen University, Health Science Center, Shenzhen Second People's Hospital, Shenzhen 518037, China

4 Department of Radiology, Wayne State University, Detroit, MI 48201, USA

5 Sino-Dutch Biomedical and Information Engineering School of Northeastern University, Shenyang, China 


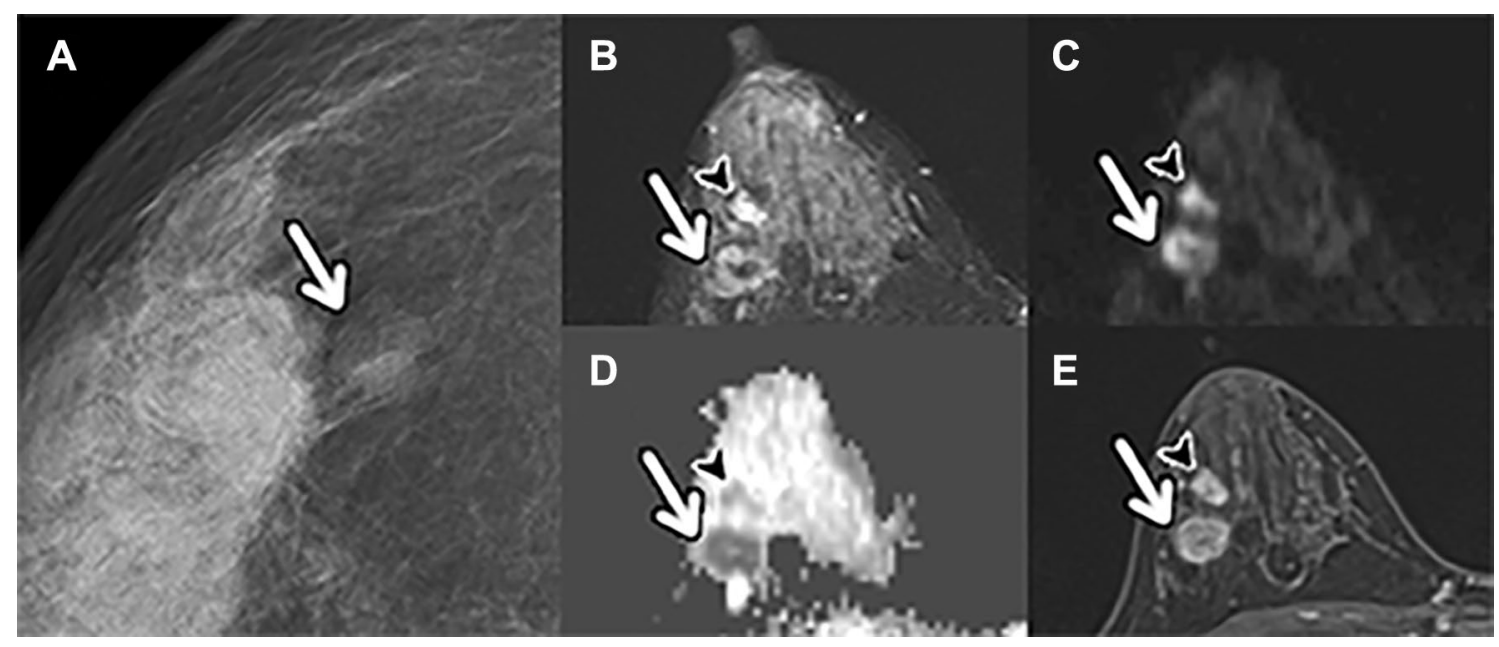

Fig. 4 A 38-year-old woman with dense breasts, with pathologically proven invasive carcinoma of no special type and mucinous carcinoma in the right breast. The lesion visibility scores of MG, DWI + TIRM, and clinical MRI of the lesion (arrow) were 4, 5, and 5, respectively. The breast cancer detection scores of MG, DWI+ TIRM, and clinical MRI of the lesion (arrow) were 3, 4, and 5, respectively. The lesion visibility scores of MG, DWI+ TIRM, and clinical MRI of the lesion (arrow head) were 2, 5, and 5, respectively. The breast cancer detection scores of MG, DWI+ TIRM, and clinical MRI of the

Publisher's Note Springer Nature remains neutral with regard to jurisdictional claims in published maps and institutional affiliations. lesion (arrow head) were 2, 4, and 5, respectively. a MG showed a high-density lesion with a circumscribed margin. b TIRM and $\mathbf{c}$ DWI $\left(b=0.8 \times 10^{-3} \mathrm{~s} / \mathrm{mm}^{2}\right)$ demonstrated a heterogeneous high-signalintensity lesion (arrow) and a homogeneous high-signal-intensity lesion (arrow head) in the right breast. d ADC values of these two lesions were $0.78 \times 10^{-3} \mathrm{~mm}^{2} / \mathrm{s}$ (arrow) and $0.83 \times 10^{-3} \mathrm{~mm}^{2} / \mathrm{s}$ (arrow head). e Contrast-enhanced MRI showed both lesion heterogeneous enhancement 